\title{
Sources of beta cells inside the pancreas
}

\author{
Sofie De Groef ${ }^{1} \cdot$ Willem Staels $^{1}$ - Naomi Van Gassen ${ }^{1} \cdot$ Marie Lemper $^{1} \cdot$ Yixing Yuchi $^{1}$ • \\ Mozhdeh Sojoodi $^{1}$ • Leen Bussche ${ }^{1}$ - Yves Heremans ${ }^{1}$ - Gunter Leuckx ${ }^{1}$ - Nico De Leu ${ }^{1}$. \\ Mark Van de Casteele ${ }^{1} \cdot$ Luc Baeyens $^{1} \cdot$ Harry Heimberg ${ }^{1}$
}

Received: 12 November 2015 / Accepted: 16 December 2015/Published online: 6 April 2016

(C) Springer-Verlag Berlin Heidelberg 2016

\begin{abstract}
The generation of beta(-like) cells to compensate for their absolute or relative shortage in type 1 and type 2 diabetes is an obvious therapeutic strategy. Patients first received grafts of donor islet cells over 25 years ago, but this procedure has not become routine in clinical practice because of a donor cell shortage and (auto)immune problems. Transplantation of differentiated embryonic and induced pluripotent stem cells may overcome some but not all the current limitations. Reprogramming exocrine cells towards functional beta(-like) cells would offer an alternative abundant and autologous source of beta(-like) cells. This review focuses on work by our research group towards achieving such a source of cells. It summarises a presentation given at the 'Can we make a better beta cell?' symposium at the 2015 annual meeting of the EASD. It is accompanied by two other reviews on topics from this symposium (by Amin Ardestani and Kathrin Maedler, DOI: 10.1007/s00125016-3892-9, and by Heiko Lickert and colleagues, DOI: 10. 1007/s00125-016-3949-9) and a commentary by the Session Chair, Shanta Persaud (DOI: 10.1007/s00125-016-3870-2).
\end{abstract}

Keywords Beta cells $\cdot$ Diabetes $\cdot$ Exocrine pancreas $\cdot$ Growth factors $\cdot$ Injury $\cdot$ Macrophages $\cdot$ Proliferation $\cdot$ Regeneration · Review $\cdot$ Transcription factors $\cdot($ Trans)differentiation

\section{Abbreviations \\ CNTF Ciliary neurotrophic factor \\ E Embryonic day}

Harry Heimberg

Harry.Heimberg@vub.ac.be

1 Diabetes Research Center, Vrije Universiteit Brussel, Laarbeeklaan 103, B1090 Brussels, Belgium

\author{
EGF Epidermal growth factor \\ $\mathrm{ER} \alpha \quad$ Oestrogen receptor $\alpha$ \\ LIF Leukaemia inhibitory factor \\ M-CSFR Macrophage colony-stimulating factor receptor \\ MAPK Mitogen-activated protein kinase \\ MS MAPK and STAT3 \\ NGN3 Neurogenin 3 \\ PDL Partial duct ligation \\ STAT3 Signal transducer and activator of transcription 3
}

Shifts in differentiated cell fate, also referred to as transdifferentiation, are generally rare, but spark curiosity in many cell biologists. Although experimental evidence for the basic concept of this phenomenon was provided long ago $[1,2]$, it is only over the last decade that it has appeared centrestage of cell therapy design $[3,4]$. This short overview focuses on our own achievements in the area of pancreas cell transdifferentiation and the generation of beta cells, and discusses their potential impact on the development of potential diabetes therapies (see also [5]) (Fig. 1).

Fifteen years ago we started to evaluate the possibility of human pancreatic exocrine-to-beta cell reprogramming [6]. Duct cells of adult human pancreas were transduced with recombinant adenoviral vectors that constitutively expressed neurogenin 3 (NGN3), a basic helix-loop-helix transcription factor essential for development of the endocrine pancreas [7, 8]. Ectopic expression of Neurog3 induced a number of key proteins for beta cell formation and function, including insulin, albeit in few cells and at a level far insufficient to be clinically relevant [6]. Indeed, genome-wide transcriptome analysis showed that less than $10 \%$ of full duct to endocrine cell conversion was achieved [9]. Co-expression of Pdx1, Ngn3 and MafA, a combination shown to transdifferentiate exocrine to beta 
Fig. 1 Schematic overview of our own research into pancreas cell transdifferentiation and the generation of beta cells, as described in the main text. $\mathrm{LeMS}^{\mathrm{CA}}$, lentivirus-mediated expression of constitutively active MAPK and STAT3 (MS)
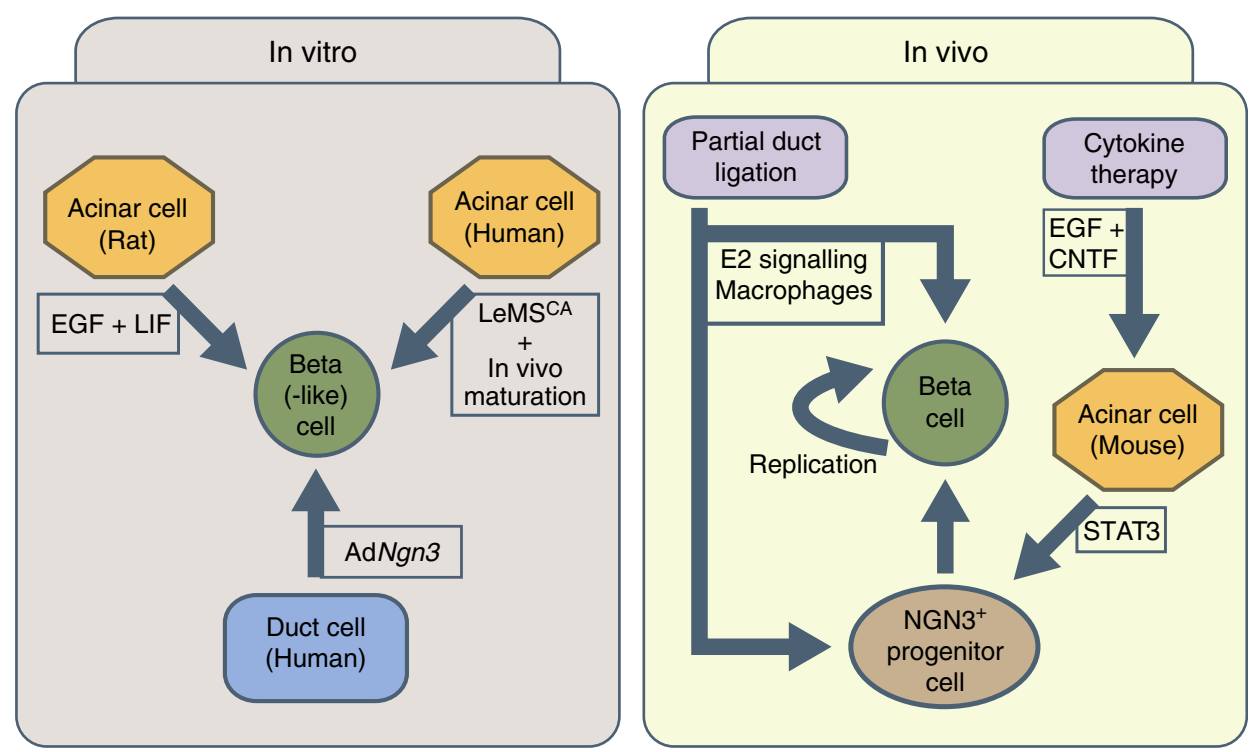

cells when applied to mouse pancreas [10,11], delivered no improvement over Ngn3 alone after transduction into adult human pancreatic duct cells [9].

To gain more insight into the cells and factors needed for pancreas cell transdifferentiation, we used two experimental models: partial duct ligation (PDL) [12, 13] and growth factor therapy [14].

\section{Partial duct ligation to identify cells and signals for beta cell generation}

PDL is a microsurgical technique [15] in which the main pancreatic duct is ligated, whereafter acinar cells disappear and both beta and alpha cell mass doubles. Endocrine cell generation results from the differentiation of non-beta cells (facultative progenitor cells) and proliferation of pre-existing and newly formed endocrine cells $[12,13,16]$. Stressful conditions caused by severe tissue damage such as PDL may thus increase cell plasticity and result in the interconversion of differentiated cell types (reviewed in [17]). In part due to the complexity of the surgical technique, outcome variability complicates the model, leaving room for controversy (reviewed in [18]). However, confirmation of our data by supplementary techniques, including optical projection tomography and by independent scientists encouraged us to further dissect the signals that drive endocrine cell generation in PDL pancreas. Given the massive recruitment of immune cells (especially monocytes and macrophages) to the PDL pancreas, and because macrophages have been shown to promote beta cell proliferation in PDL pancreas [19], we assessed their diversity and the factors driving myeloid cell dynamics in normal and PDL pancreas [20]. While tissueresident MHC class $\mathrm{II}^{+}$macrophages predominated in naive and sham-operated pancreas, PDL induced massive monocyte infiltration followed by macrophage differentiation and maturation. Monocyte recruitment was dependent on C-C chemokine receptor 2 (CCR2) and macrophage colony- stimulating factor receptor (M-CSFR), as well as on the local proliferation of macrophages under the influence of M-CSFR signalling. Tissue-resident macrophages rather than monocyte-derived ones produced beta cell mitogens. Continued research on macrophage function in pancreas may thus uncover the mechanisms underlying their roles in beta cell protection during pancreatitis, the enhancement of beta cell activity and their contribution to the control of beta cell proliferation and regeneration following beta cell injury (reviewed in [21]). The identification and characterisation of macrophage subtypes and their secretome might contribute to the development of new therapeutic strategies for diabetes. The possibility that myeloid cells other than macrophages and/or lymphoid cells play a role in the regulation of beta cell proliferation and facultative progenitor cell activation cannot be excluded.

While performing cell lineage tracing studies in PDL pancreas using tamoxifen-mediated induction of fluorescent reporter gene expression in (promotor-defined) specific cell populations, we observed a strong reduction in beta cell proliferation as compared with mice that did not receive tamoxifen [22]. When applied to pregnant mice, which show elevated beta cell proliferation to meet the increased insulin needs (reviewed in [23]), tamoxifen blocked beta cell cycling and thus antagonised oestrogen signalling. Loss- and gain-of-function studies confirmed the role of oestrogen signalling for beta cell proliferation in PDL pancreas. Indeed, administration of oestradiol to increase circulating levels further increased beta cell proliferation, while injection of aromasin, an inhibitor of aromatase that catalyses the rate-limiting step of oestradiol synthesis, blunted the PDL-induced increase in beta cell proliferation. Moreover, nuclear translocation of oestrogen receptor $\alpha(\mathrm{ER} \alpha)$ was 
prevented by tamoxifen-treatment, and $E s r 1^{-/}$mice showed no increase in beta cell proliferation following PDL [22]. Oestradiol levels were below the detection limit in shamoperated male mice but were clearly present in the circulation of male mice with PDL pancreas. Preliminary data point to adipose tissue as a principal oestrogen source. Indeed, a distinct population of autofluorescent cells with low density that stained with Oil Red O could be isolated from PDL pancreas but not from naive or sham-operated pancreas. In addition, adipocytespecific transcripts, such as those for adiponectin, leptin and fatty acid synthase, were highly abundant in the ligated part of the pancreas and were strongly reduced by tamoxifen (Y. Yuchi, M. Van de Casteele, H. Heimberg, unpublished data). Importantly, we also analysed the influence of ER $\alpha$ signalling during the development of the endocrine pancreas, at embryonic day (E)15.5 and E18.5, when endocrine progenitor activation peaks and the proliferation rate of newly formed beta cells is high, respectively. Not only was the number of $\mathrm{NGN}^{+}$endocrine progenitor cells considerably decreased by tamoxifen and ESR1 knockout, so was beta cell proliferation. We therefore concluded that $\mathrm{ER} \alpha$ signalling regulates beta cell formation during pancreas development and following injury [22] and we are currently investigating the beta cell specificity of the above-mentioned processes using mice with beta cell-specific deletion of Esrl. If our investigations are successful, we will examine whether oestrogen conjugated to glucagon-like peptide-1 (GLP-1) to allow targeted delivery to beta cells [24] is able to specifically (hyper)stimulate beta cell proliferation in PDL pancreas and of engrafted human beta cells.

\section{Growth factor therapy for beta cell generation}

Because rat acinar cells can be transdifferentiated into functional beta(-like) cells when exposed to epidermal growth factor (EGF) and leukaemia inhibitory factor (LIF) [25], a mouse model was developed to investigate whether this in vitro finding is translatable to mice with long-term hyperglycaemia. Mice that were injected with alloxan, a relatively beta cell-specific toxin, rapidly became hyperglycaemic. Four weeks later, when the mice had been diabetic for one-third of their lifetime, a mini-osmotic pump containing EGF and ciliary neurotrophic factor (CNTF) was implanted into the peritoneum. Like LIF, CNTF is a member of the IL-6 family of growth factors, but unlike LIF, it did not cause an elevated mortality rate. EGF and CNTF were released from the pump during 1 week and, within this period, glycaemia significantly decreased in $65 \%$ of mice [14]. The speed and efficiency of the process are remarkable, but it still is unclear why $35 \%$ of the mice did not respond to the combination of EGF and CNTF. Based on careful cell-specific lineage tracing and cell-specific loss-of-function experiments, we concluded that EGF and CNTF can restore glycaemia to near normal levels for an extended period of time in mice with chronic hyperglycaemia, mainly by cell-autonomous signal transducer and activator of transcription 3 (STAT3)-mediated acinar cell transdifferentiation but also through an intermediary cell type in which $\mathrm{Ngn} 3$ (re-)expression is pivotal [14]. Interestingly, the crucial role of NGN3 in this regeneration process suggests the dedifferentiation of a subpopulation of acinar cells, possibly towards a facultative beta cell progenitor phenotype. Increased knowledge of the epigenetic changes that control the transdifferentiation of acinar cells into beta-like cells will be needed if potential therapies are to be developed, since the two growth factors have associated side effects. Moreover, any potential drugs should optimise the efficiency of the process.

To provide a proof of concept with regard to the translation of growth factor therapy from mice to man, human exocrine pancreas cells were exposed to EGF and LIF or CNTF. Unfortunately, there was no shift in their differentiation status towards endocrine cells. Because we hypothesised that the ligands were not able to signal, and because intracellular signalling by EGF and CNTF occurs through mitogen-activated protein kinase (MAPK) and STAT3 (MS), adult human exocrine cells were transduced with recombinant lentiviral vectors that expressed constitutively active forms of MS ( $\left.\mathrm{MS}^{\mathrm{CA}}\right)$. Interestingly, 1 week after transduction, $40 \%$ of the $\mathrm{MS}^{\mathrm{CA}}$-transduced exocrine cells expressed high levels of NGN3, while only few contained insulin [26]. When these cells were grafted under the kidney capsule of immunocompromised mice for 42 days, the number of insulin-positive cells slightly increased, while NGN3 completely disappeared. However, transplantion beyond 90 days resulted in increasing amounts of circulating human C-peptide. Since human beta(-like) cells are insensitive to alloxan as a result of low or absent expression of glucose transporter type 2 [27], treatment of these mice with alloxan resulted in ablation of the endogenous beta cells, while the human beta(-like) cells of the graft were spared. As a result, there was a rapid increase in the blood sugar level that was countered by a sudden boost of human insulin released by the grafted cells [26]. Research is ongoing to explore the use of alternatives for virus-mediated delivery of MS ${ }^{\mathrm{CA}}$ to exocrine cells following unlimited expansion. Since the expansion protocol selects for adult bipotent facultative progenitors in the mouse [28] but also in humans (W. Staels, Y. Heremans, H. Heimberg, unpublished data), their translational potential for routine transplantation in diabetes patients warrants further investigation.

Acknowledgements Our work is financially supported by the VUB Research Council, the Institute for the Promotion of Innovation by Science and Technology in Flanders (IWT), the Beta Cell Biology Consortium, Stichting Diabetes Onderzoek Nederland, the Fund for 
Scientific Research-Flanders (FWO), the European Union Seventh Framework Program and the Belgian Science Policy.

Duality of interest The authors declare that there is no duality of interest associated with this manuscript.

Contribution statement All authors contributed to the collection of data the article is based upon, critically revised the text and approved the version to be published.

\section{References}

1. Gurdon JB, Elsdale TR, Fischberg M (1958) Sexually mature individuals of Xenopus laevis from the transplantation of single somatic nuclei. Nature 182:64-65

2. Davis RL, Weintraub H, Lassar AB (1987) Expression of a single transfected cDNA converts fibroblasts to myoblasts. Cell 51:9871000

3. Slack JM (2007) Metaplasia and transdifferentiation: from pure biology to the clinic. Nat Rev Mol Cell Biol 8:369-378

4. Vierbuchen T, Wernig M (2011) Direct lineage conversions: unnatural but useful? Nat Biotechnol 29:892-907

5. Staels W, Heremans Y, Heimberg H (2015) Reprogramming of human exocrine pancreas cells to beta cells. Best Pract Res Clin Endocrinol Metab 29:849-857

6. Heremans Y, Van De Casteele M, in't Veld P et al (2002) Recapitulation of embryonic neuroendocrine differentiation in adult human pancreatic duct cells expressing neurogenin 3. J Cell Biol 159:303-312

7. Apelqvist A, Li H, Sommer L et al (1999) Notch signalling controls pancreatic cell differentiation. Nature 400:877-881

8. Gradwohl G, Dierich A, LeMeur M, Guillemot F (2000) Neurogenin 3 is required for the development of the four endocrine cell lineages of the pancreas. Proc Natl Acad Sci U S A 97:16071611

9. Swales N, Martens GA, Bonne S et al (2012) Plasticity of adult human pancreatic duct cells by neurogenin3-mediated reprogramming. PLoS One 7, e37055

10. Zhou Q, Brown J, Kanarek A, Rajagopal J, Melton DA (2008) In vivo reprogramming of adult pancreatic exocrine cells to betacells. Nature 455:627-632

11. Li W, Cavelti-Weder C, Zhang Y et al (2014) Long-term persistence and development of induced pancreatic beta cells generated by lineage conversion of acinar cells. Nat Biotechnol 32:1223-1230

12. Xu X, D'Hoker J, Stange G et al (2008) Beta cells can be generated from endogenous progenitors in injured adult mouse pancreas. Cell 132:197-207
13. Van de Casteele M, Leuckx G, Baeyens L et al (2013) Neurogenin $3+$ cells contribute to beta-cell neogenesis and proliferation in injured adult mouse pancreas. Cell Death Dis 4, e523

14. Baeyens L, Lemper M, Leuckx G et al (2014) Transient cytokine treatment induces acinar cell reprogramming and regenerates functional beta cell mass in diabetic mice. Nat Biotechnol 32:76-83

15. De Groef S, Leuckx G, Van Gassen N, et al (2015) Surgical injury to the mouse pancreas through ligation of the pancreatic duct as a model for endocrine and exocrine reprogramming and proliferation. J Vis Exp e52765

16. Cai Y, Yuchi Y, De Groef S et al (2014) IL-6-dependent proliferation of alpha cells in mice with partial pancreatic-duct ligation. Diabetologia 57:1420-1427

17. Erler P, Monaghan JR (2015) The link between injury-induced stress and regenerative phenomena: a cellular and genetic synopsis. Biochim Biophys Acta 1849:454-461

18. Van de Casteele M, Leuckx G, Cai Y et al (2014) Partial duct ligation: beta-cell proliferation and beyond. Diabetes 63:25672577

19. Xiao X, Gaffar I, Guo P et al (2014) M2 macrophages promote beta-cell proliferation by up-regulation of SMAD7. Proc Natl Acad Sci U S A 111:E1211-E1220

20. Van Gassen N, Van Overmeire E, Leuckx G et al (2015) Macrophage dynamics are regulated by local macrophage proliferation and monocyte recruitment in injured pancreas. Eur J Immunol 45:1482-1493

21. Van Gassen N, Staels W, Van Overmeire E et al (2015) Concise review: macrophages: versatile gatekeepers during pancreatic $\beta$ cell development, injury, and regeneration. Stem Cells Transl Med 4:555-563

22. Yuchi Y, Cai Y, Legein B et al (2015) Estrogen receptor $\alpha$ regulates $\beta$-cell formation during pancreas development and following injury. Diabetes 64:3218-3228

23. Rieck S, Kaestner KH (2010) Expansion of beta-cell mass in response to pregnancy. Trends Endocrinol Metab TEM 21:151-158

24. Finan B, Yang B, Ottaway N et al (2012) Targeted estrogen delivery reverses the metabolic syndrome. Nat Med 18:1847-1856

25. Baeyens L, De Breuck S, Lardon J, Mfopou JK, Rooman I, Bouwens L (2005) In vitro generation of insulin-producing beta cells from adult exocrine pancreatic cells. Diabetologia 48:49-57

26. Lemper M, Leuckx G, Heremans Y et al (2015) Reprogramming of human pancreatic exocrine cells to beta-like cells. Cell Death Differ 22:1117-1130

27. Eizirik DL, Pipeleers DG, Ling Z, Welsh N, Hellerstrom C, Andersson A (1994) Major species differences between humans and rodents in the susceptibility to pancreatic beta-cell injury. Proc Natl Acad Sci U S A 91:9253-9256

28. Huch M, Bonfanti P, Boj SF et al (2013) Unlimited in vitro expansion of adult bi-potent pancreas progenitors through the Lgr5/Rspondin axis. EMBO J 32:2708-2721 